\title{
Anatomy and relationships of the fuxianhuiid euarthropod Guangweicaris from the early Cambrian Guanshan Biota in Kunming, Yunnan, Southwest China revisited
}

Yichen Wu and Jianni Liu

Acta Palaeontologica Polonica 64 (3), 2019: 543-548 doi:https://doi.org/10.4202/app.00542.2018

The Guanshan Biota (Cambrian Series 2, Stage 4) is a typical Burgess Shale-type biota, and is one of the most significant Cambrian Konservat-Lagerstätten from China, in addition to the well-known Chengjiang and Kaili biotas. Guangweicaris is a relatively common arthropod from the Guanshan Biota. Based on new specimens from the Longbaoshan and Xinglongcun sections of the Wulongqing Formation in Kunming, we herein report novel features of Guangweicaris spinatus, such as the eye-bearing anterior sclerite that is associated with the head shield, as well as revised details of the trunk, including the ventral appendages. A detailed comparison of Guangweicaris, Fuxianhuia and Shankouia suggests a close affinity between Guangweicaris and Fuxianhuia, which improves our understanding of the evolution of early Cambrian euarthropods.

Yichen Wu [wuyicheneli@126.com] and Jianni Liu [liujianni@126.com], State Key Laboratory of Continental Dynamics, Shaanxi Key Laboratory of Early Life and Environment, Department of Geology, Northwest University, Xi'an, China.

This is an open-access article distributed under the terms of the Creative Commons Attribution License (for details please see creativecommons.org), which permits unrestricted use, distribution, and reproduction in any medium, provided the original author and source are credited. 